\title{
Horizons/Théâtre
}

Revue d'études théâtrales

\section{Le théâtre estudiantin à la recherche d'une gloire perdue}

\section{Fernand Nouwligbèto}

\section{(2) OpenEdition}

1 Journals

Édition électronique

URL : https://journals.openedition.org/ht/1206

DOI : 10.4000/ht.1206

ISSN : 2678-5420

Éditeur

Presses universitaires de Bordeaux

\section{Édition imprimée}

Date de publication : 31 décembre 2018

Pagination : 118-135

ISBN : 979-10-300-0318-5

ISSN : 2261-4591

\section{Référence électronique}

Fernand Nouwligbèto, « Le théâtre estudiantin à la recherche d'une gloire perdue », Horizons/Théâtre [En ligne], 13 | 2018, mis en ligne le 01 janvier 2019, consulté le 17 mai 2022. URL : http:// journals.openedition.org/ht/1206 ; DOI : https://doi.org/10.4000/ht.1206

\section{cc) $(1) \odot$}

La revue Horizons/Théâtre est mise à disposition selon les termes de la Licence Creative Commons Attribution - Pas d'Utilisation Commerciale - Pas de Modification 4.0 International. 


\section{FERNAND NouWLIGBèto}

Enseignant à l'Université d'Abomey-Calavi au Bénin, Fernand Nouwligbèto est l'auteur de plusieurs articles scientifiques, publiés dans diverses revues en Afrique et en Europe, et d'un essai intitulé Théâtre béninois : logiques marchandes et enjeux esthétiques (Cotonou, CIREF, 2017). Ses domaines de recherche sont les arts du spectacle vivant, en général, et le théâtre, en particulier. Romancier (La Foudre sous scellés, Cotonou, Aziza, 2003) et dramaturge (Zongo Giwa de la forêt déviergée, Paris, L'Harmattan, 2005 ; Roi LMD International, Cotonou, Perma, 2018), il est membre du Centre international de recherches et d'études francophones et président du Conseil d'administration de l'association culturelle Perma Sillons d'Or (Persildor).

Mail: fnouwligbeto@gmail.com

Résumé : Quels sont les déterminants de la léthargie profonde dans laquelle semble plongé, depuis 1995, l'Ensemble artistique et culturel des Étudiants (EACE) de I'Université d'Abomey-Calavi ? L'objectif de la présente étude est d'élucider les causes du crépuscule artistique de l'une des troupes les plus célèbres du théâtre béninois, créée depuis 1977. Nous présumons que l'autocensure inhibe les élans artistiques des étudiants en même temps qu'elle induit à leur niveau des stratégies de captage de rente. Sur la base des outils de la critique

Abstract : What are the determinants of the deep lethargy in which seems to be plunged, since 1995, the "Ensemble artistique et culturel des Étudiants" (EACE) of the University of Abomey-Calavi? The objective of this study is to elucidate the causes of the artistic twilight of one of the most famous troupes in the Beninese theater, created since 1977. We presume that self-censorship inhibits the artistic impulses of students as well as it induces rent collection strategies at their level. On the basis of the tools of historical criticism, sometimes combined with those historique, combinée parfois avec ceux de I'approche "bourdieusienne " des champs littéraires, la vérification de cette hypothèse s'est faite à travers un développement en quatre articulations : après un aperçu sur le contexte de création de l'E.ACE, puis une présentation schématique de cette troupe estudiantine, l'attention a été portée sur l'évolution de la production dramatique de celle-ci avant que ne soit posé le diagnostic de la crise à laquelle elle est confrontée.

Mots-cLÉs : théâtre, crise, champ littéraire, horizon d'attente, stratégies.

of the "bourdieusian" approach to literary fields, the verification of this hypothesis was made through a development in four joints: after an overview on the context of creation of EACE then a schematic presentation of this student troupe, attention was paid to the evolution of the dramatic production of this artistic complex before the diagnosis of the crisis it was faced was made.

KEYWORDS: theater, crisis, literary field, expectation outlook, strategies. 


\section{Le théâtre estudiantin à la recherche d'une gloire perdue}

\section{Introduction}

ENTRe 1977 et 1995, LE THÉÂTRE DES ÉTUdiANTS DE L'UNIVERSitÉ D’AвomeY-CALAVI a redonné de la vigueur à la création dramatique béninoise d'expression française. L'Ensemble artistique et culturel des Étudiants (EACE), créé depuis 1977, a su chaque fois soulever un enthousiasme délirant autour de ses spectacles grâce à l'audace novatrice de ceux-ci, pour la plupart marqués par l'esthétique de la création collective.

Mais depuis la seconde moitié des années 1990, une chape de plomb semble progressivement étouffer l'inspiration des étudiants, alors même que d'autres troupes estudiantines ont vu le jour et que le paysage théatral au Bénin a enregistré des avancées telles que la naissance de structures de formation en art dramatique et l'existence de structures publiques et privées d'appui à la création. Cette léthargie suscite des questionnements sur les facteurs explicatifs de sa survenue et ses implications thématiques et esthétiques. L'objectif de l'étude étant d'élucider les causes de ce crépuscule artistique, il apparaît que l'auto-censure inhibe les élans artistiques des étudiants en même temps qu'elle induit à leur niveau des stratégies de captage de rente.

La vérification de ce postulat s'est principalement faite sur la base de la critique historique, une approche pour laquelle le contexte de l'œuvre, le milieu de vie de l'auteur et la périodisation sont des notions essentielles dans l'appréhension du fait littéraire ou artistique. Toutefois, il a été aussi parfois fait recours à la théorie « bourdieusienne » du champ littéraire, ce concept s'appréhendant « comme un espace de concurrence dans lequel s'affrontent, en vue d'acquérir un profit matériel et/ou symbolique, des producteurs dont les positions, à un moment donné de l'histoire du champ, dépendent à la fois de leurs dispositions initiales et de la pertinence de leurs prises de position ${ }^{1} \gg$. Appliquées à l'histoire de l'EACE et à son palmarès, ces approches ont permis de construire un développement en quatre axes : après un aperçu sur le 
contexte de création de l'EACE, puis une présentation schématique de cette troupe estudiantine, l'attention a été portée sur l'évolution de sa production dramatique avant que ne soit posé le diagnostic de la crise à laquelle elle est confrontée.

\section{Contexte de création de l'EACE}

Le contexte de création de l'EACE est étudié d'un double point de vue, aussi bien sur le plan de l'environnement socio-politique que sur le plan culturel.

\section{Un environnement sociopolitique marqué par l'apogée du régime marxiste-léniniste}

L'EACE est né en 1977, soit cinq ans après l'arrivée au pouvoir, le 26 octobre 1972, de la junte militaire dénommée « Gouvernement Militaire Révolutionnaire » (GMR). Ces jeunes hommes en armes avaient planifié et réussi un coup d'État destiné, selon eux, à mettre un terme à l'instabilité sociopolitique de la république du Dahomey, ancienne colonie française située en Afrique de l'Ouest, précisément sur la côte atlantique, entre le Nigéria à l'est et le Togo à l'ouest, le Burkina Faso au nord-ouest et le fleuve Niger au nord-est. Du $1^{\text {er }}$ août 1960, année de son indépendance, au 25 octobre 1972, cet État étendu sur $114763 \mathrm{~km}^{2}$ avait vécu au gré des rivalités ethnico-régionalistes et d'une douzaine au moins de putschs réussis. Aussi, pour les jeunes révolutionnaires du 26 octobre 1972, fallait-il en finir une fois pour toutes avec la gabegie en instaurant un pouvoir fort, gage selon eux de l'unité nationale et du développement économique. Dans le discours-programme du 30 novembre 1972, prononcé par Mathieu Kérékou, le nouvel homme fort du pays, les grandes orientations du développement national étaient claires :

Il s'agira de : - liquider définitivement l'ancienne politique à travers les hommes, les structures et l'idéologie qui la portent. Comptons d'abord sur nos propres forces, sur nos propres ressources, sur l'initiative créatrice des larges masses dans notre lutte pour nous libérer de la domination étrangère, pour développer notre économie et pour donner à notre peuple la dignité et la personnalité d'un peuple libre; - réorganiser toute la structure économique, culturelle et sociale de notre pays dans le sens d'une libération de la domination étrangère, d'une éradication de la corruption, de la concussion et du népotisme, et d'une plus grande efficacité; - développer nos relations extérieures avec tous les pays sans discrimination sur la base du respect de la souveraineté nationale, de l'égalité et de l'avantage réciproque ; - affermir l'autorité de l'État ${ }^{2}$. 
Une lecture attentive de cet extrait, précisément l'analyse du champ sémantique du nationalisme dahoméen («liquider », « comptons sur nos propres forces $\gg$, « domination étrangère $\gg . .$.$) dans le contexte mondial de la guerre$ froide, révèle déjà les grandes options idéologiques vers lesquelles s'orientera le GMR. De ce fait, le 30 novembre 1974, l'État adopte le socialisme scientifique comme voie de développement et le marxisme-léninisme comme guide dans la mise en œuvre des actions publiques. En mars 1975, un arrêté du ministre en charge de l'Information et de l'Orientation nationale subordonne à une autorisation préalable tout « écrit politique sur la Révolution ${ }^{3}$ ». En août, le ministre de l'Intérieur fait diffuser par les médias un communiqué qui énonce ceci :

l'organisation de récitals, de chansons, de poésies et de représentations théâtrales, artistiques, culturelles et folkloriques sur toute l'étendue de Territoire National est soumise à l'autorisation du ministre de l'Intérieur et de la Sécurité, après avis du ministre de la Jeunesse, de la Culture Populaire et des Sports ${ }^{4}$.

Enfin, le 30 novembre 1975, Mathieu Kérékou et son gouvernement changent le nom du pays et ses attributs : le Dahomey est devenu République populaire du Bénin et le drapeau tricolore (vert, jaune, rouge) a cédé la place à un autre, bicolore (un fond rectangulaire vert frappé en haut, au coin gauche, d'une étoile rouge). Le monopartisme a été instauré et le Parti de la Révolution populaire du Bénin (PRPB) fut le seul parti politique autorisé.

Cette radicalisation progressive du régime eut donc comme corollaires une restriction des champs de liberté, une obligation imposée à tous les Béninois d'être membre du PRPB et un glissement sournois vers le règne de la pensée unique. Cela eut des conséquences sur l'environnement socio-culturel qui, placé sous le contrôle de l'État, était malgré tout assez dynamique.

\section{Un environnement socio-culturel dynamique}

L'EACE n'est pas né dans un paysage culturel vierge. Avant sa création, les initiatives artistiques ne manquaient pas.

Héritiers de riches traditions culturelles et du théâtre «pontin », animé dans les années $1930^{5}$ entre autres par le collectif des élèves dahoméens de l'École normale William-Ponty du Sénégal, les Dahoméens ont toujours pratiqué l'art dramatique. Au lendemain de l'indépendance, en août 1960, des auteurs comme Julien Alapini (Acteurs noirs) et Jean Pliya (Kondo le requin, La Secrétaire particulière) ont publié des pièces théâtrales, tout comme le fera, bien plus tard, Sévérin Akando (Bio Guera et le destin d'un peuple, Le mariage de Sika n'aura pas lieu). 
Plus dynamique, le théâtre oral dahoméen a acquis, au plan national comme international, une notoriété certaine grâce aux prestations de nombreuses troupes privées ou publiques, telles que les Ballets Africains du Dahomey appelés « Egblemakou » d'Édouard Gaye, les Muses de Boniface Makponsè et l'Ensemble national folklorique du Dahomey dirigé par Flavien Campbell. En 1962, cet Ensemble a été la toute première troupe non européenne à remporter le Challenge au Théâtre des Nations à Paris. Non moins illustres ont été la Troupe du Théâtre de l'IRAD (Institut de recherches appliquées du Dahomey) et Les Cerveaux noirs, toutes deux dirigées par Lazare Houétin, ancien élève du lycée Victor Ballot à Porto-Novo.

Revenu de ses études à l'Université de Vincennes à Paris, Lazare Houétin a apporté une contribution déterminante au théâtre béninois à travers la création de nombreux spectacles, la formation des comédiens, l'animation de centres culturels comme la Société d'exploitation des centres de spectacles, etc. Il a été l'un des principaux artisans de la création de l'Ensemble artistique polyvalent Zama-Hara, en février 1975. De même, c'est lui qui fit découvrir à Paul Ayémona, le futur fondateur de l'EACE, les trésors de la création artistique.

\section{L'EACE, une initiative privée audacieuse}

La naissance de l'EACE a été le fruit d'un singulier concours de circonstances. En 1974, le Dahomey se préparait à participer au Festival international de la jeunesse francophone, encore appelé « Superfrancofête », prévu pour se dérouler la même année au Québec, au Canada, du 13 au 24 août. Dans ce cadre, l'État avait mis sur pied un comité de sélection des artistes devant former, à l'occasion, la troupe nationale. Constitué d'hommes de la scène et d'intellectuels comme Lazare Houétin, Koffi Gahou et Jérôme Carlos, le comité a parcouru les six provinces du pays. Dans le Borgou, il rencontra les artistes à Parakou, ville située à $400 \mathrm{~km}$ au nord de Cotonou. Parmi les candidats, Lazare Houétin et ses collègues sélectionnèrent un jeune élève du lycée Mathieu Bouké appelé Paul Ayémona.

Celui-ci eut alors le loisir de suivre tout le processus de création de la pièce théâtrale Houénoussou, que le Dahomey présenta au Festival de Québec ${ }^{6}$. Ébloui par cette participation à un événement culturel international, Paul Ayémona s'engagea davantage dans l'animation du paysage culturel. Après l’obtention de son baccalauréat, il intégra l'Université nationale du Bénin, créée depuis en 1970. Dès lors, il n'eut plus qu'un rêve : donner libre cours à sa passion et allumer la même ferveur artistique dans le cœur de ses ca- 
marades. Certes, il y avait déjà sur le campus universitaire quelques activités culturelles, organisées ici et là par des étudiants. Mais il manquait une structure formelle et solide, qui puisse fédérer les différentes initiatives. Dans l'interview accordée au journal Canal de l'Art, Paul Ayémona éclaire sur les circonstances de création de l'EACE :

J'ai eu toujours du mal à répondre à cette question. En effet la création de l'EACE n'est pas le fait [sic] de penser et de décider d'un individu; mais la résultante de plusieurs circonstances et aussi bien [que, NDA] l'engagement de personnes. Il est bon de rappeler qu'en septembre 1976, nous étions en pleine période révolutionnaire. Il fallait changer le régime scolaire et universitaire. L'année scolaire devrait débuter en janvier pour prendre fin en juin. Alors qu'il fallait occuper les élèves et étudiants entre septembre et janvier. Ce temps était appelé « période transitoire $\gg$. Pendant ce temps, les élèves devaient s'occuper de « production 》. Certains amis et moi avons pensé que l'art et la culture constituent également de la production. Nous avons osé avec les parcelles [de terrain] qui nous étaient affectées. Nous nous retrouvions à l'amphi FLASH pour faire des entraînements de théâtre, de chants... C'était les prémices qui ont vu jour en décembre 1976 après un séminaire au lycée agricole de Sékou dont [sic] le nom: Ensemble Artistique et Culturel des Étudiants ${ }^{7}$.

Le nom de baptême de cette nouvelle association culturelle était certainement inspiré à Paul Ayémona et à ses jeunes camarades, dont Orou N'morou, Darius Awowedji, etc., par les dénominations de la plupart des autres troupes, notamment l'Ensemble national folklorique du Dahomey et l'Ensemble artistique polyvalent Zama-Hara. Toutefois, l'EACE se distinguait de ceux-ci par son statut d'association universitaire et par d'autres caractéristiques qu'il convient à présenter d'évoquer.

\section{Présentation schématique de l'EACE}

Au fil des années, l'EACE s'est doté d'organes pour assurer son fonctionnement et atteindre ses objectifs de production.

\section{Statut, administration et fonctionnement de l'EACE}

L'EACE est une troupe universitaire exclusivement animée par des étudiants de l'ex-Université nationale du Bénin, actuelle Université d'Abomey-Calavi. Elle est la branche artistique et culturelle de la Fédération nationale des Étudiants du Bénin (FNEB), puissante organisation estudiantine, à l'instar de la Fédération des étudiants de Côte d'Ivoire. Il est de notoriété publique que la FNEB a souvent été hostile à tout pouvoir exécutif en 
place. On ne peut manquer, à ce propos, de se poser ces deux questions : dans quelle mesure l'EACE, dans ses créations, reflétait-elle les prises de position sociales et politiques de la FNEB ? La crise de l'Ensemble était-elle un écho des divisions qui déchiraient également la Fédération ? Nous y reviendrons.

En tant que structure estudiantine, l'EACE est une association à but non lucratif. Elle a acquis le statut de Club Unesco, destiné à relayer les idéaux de cette institution onusienne chargée de l'Éducation, de la Science et de la Culture. L'organigramme de l'Ensemble semble avoir varié tout au long des années. Le bureau directeur actuel comprend neuf membres : deux directeurs (un directeur et un directeur adjoint), cinq secrétaires (chargés de l'administration, des finances, de l'organisation, des relations publiques...) et deux commissaires aux comptes et aux patrimoines. En quarante-deux ans, l'Ensemble a connu vingt-six directeurs, le premier étant Paul Ayémona. L'actuelle directrice, élue pour la période 2019-2020, est Cybelline de Souza.

Dès sa création en 1977, le bureau directeur a doté l'EACE de deux sections : «Théâtre et Danses nationales » et « Musique moderne ». Actuellement, l'Ensemble compte sept départements, dirigés par des directeurs de section: « Danses nationales », « Théâtre », « Musique moderne », $\ll$ Chorégraphie $\gg$, Ciné-Photo $\gg, \ll$ Coiffure $\gg$ et $\ll$ Couture $\gg$. Un principe de solidarité a toujours régi ces sections : une production à réaliser dans l'une ou l'autre amène les autres départements à s'y impliquer. Cela est surtout très visible dans la création des spectacles de théâtre dans lesquels les membres des autres sections apportent des contributions remarquables. Il s'agit, par exemple, de la confection des costumes des comédiens et de leur maquillage par les couturiers ou de l'insertion de séquences musicales dans le spectacle théâtral par les animateurs des départements « Danses », « Musique moderne » et «Chorégraphie ». Cette collaboration étroite entre les différents corps artistiques de l'Ensemble se note tout au long de la mise en œuvre du chronogramme annuel de productions.

\section{Calendrier de production}

Au fur et à mesure de son évolution, l'EACE a mis au point un calendrier de productions artistiques relativement précis auquel s'ajoutent des activités non formellement programmées. Ce calendrier, divisé en deux semestres, court de janvier à la fin de l'année civile.

Tout commence en janvier, sur le campus universitaire d'Abomey-Calavi, par la Rentrée artistique et culturelle universitaire (RACU), laquelle permet aux artistes recrutés de révéler gratuitement au public leurs productions mu- 
sicales, théâtrales et chorégraphiques. Le 14 février, à l'occasion de la célébration de la Saint-Valentin, l'Ensemble offre de nouvelles prestations aux fins de sensibiliser le monde estudiantin sur la signification de cette fête et les astuces d'une bonne gestion des relations amoureuses entre jeunes. La Journée internationale de la femme, le 8 mars, est le cadre de nouvelles sorties artistiques tandis que le mois suivant, précisément le 4 avril, l'EACE célèbre l'« Africa Dance $\gg$ à travers des spectacles de danse et de chorégraphie. En mai ou en juin, les jeunes artistes célèbrent la fête du gari, terme local désignant un produit alimentaire très populaire au Bénin et en Afrique de l'Ouest, fait à base de tubercules de manioc réduits en farine séchée, et consommé avec du sucre, des cacahuètes ou des galettes de cacahuètes. Le 11 mai, date anniversaire de la mort du célèbre chanteur Bob Marley et « Journée de la conscience nègre $\gg$, se donnent des sensibilisations artistiques sur les méfaits de la toxicomanie. Le 21 juin, l'Ensemble offre des animations diverses à l'occasion de la Fête internationale de la musique.

Le second semestre artistique commence en juillet : l'EACE, à l'instar des groupes culturels des autres universités du pays, participe aux Rencontres interuniversitaires des tambours et de la parole (RUITAP), une manifestation annuelle. En revanche, «Étincelles », le mini-festival de théâtre, est essentiellement interne : il permet à l'Ensemble de faire voir ou revoir au public quelques-unes de ses pièces. La « grande production » théâtrale est montée une fois par an après un internement de deux à quatre semaines. Le spectacle qui en est issu est souvent doublement diffusé. D’abord au plan sous-régional, l'EACE participe au Festival des Clubs Unesco des Universités d'Afrique de l'Ouest. En 1990 au Burkina Faso, 1992 au Bénin et 1993 au Mali, l'Ensemble a reçu le premier prix à ces assises. Ensuite au plan national, la troupe estudiantine initie chaque année une tournée artistique appelée «BORATA », terme forgé à partir des trois premières initiales des noms des départements du Borgou et de l'Atacora. La « grande production » est ainsi jouée dans les régions septentrionales du pays, notamment dans les chefs-lieux du Borgou, de l'Atacora, de la Donga et de l'Alibori.

En dehors de ce programme assez dense, les artistes de l'EACE répondent régulièrement à des commandes de spectacles que leur adressent souvent les autorités rectorales ou décanales à l'occasion de l'inauguration d'infrastructures, de lancement d'une nouvelle rentrée académique, d'ouvertures ou de clôtures d'événements scientifiques de grande envergure (colloque, séminaire international) ou de l'accueil d'hôtes de marque, etc. De même, l'Ensemble, depuis sa création, est parfois invité pour des tournées artistiques en Europe, en Asie et en Amérique. En 2011 par exemple, il a participé aux Rencontres 
internationales du théâtre universitaire à Liège, en Belgique. Cependant, ces invitations au plan international ont diminué au fil des ans. L'examen des productions théâtrales de l'Ensemble révèle aussi que celles-ci ont différemment évolué tout au long des années.

\section{Évolution de la production théâtrale de l'EACE}

Une esquisse de périodisation amènerait à distinguer trois grandes phases dans l'évolution de la production théâtrale de l'EACE : les années fastes (de 1977 à 1a première moitié des années 1990), les années de vaches maigres (1995 à 2009) et les années de relance timide (depuis 2010).

\section{Les années fastes (1977 à 1995)}

À propos du théâtre béninois d'expression française entre 1975 et 1990, Pierre Mèdéhouegnon fait le constat suivant :

En raison de cette mainmise de l'État et de l'orientation socialiste imposée aux artistes et aux hommes de cultures ente 1975 et 1990, le théâtre béninois de cette époque a subi le contrecoup de l'embrigadement politique dont il a été l'objet, à travers la baisse de sa qualité au profit du foisonnement de troupes de circonstances. Investi de la délicate mission de créer des cuvres de soutien à la lutte révolutionnaire et, en même temps, de promouvoir les cultures traditionnelles nationales, il s'est tout simplement sclérosé dans sa forme écrite et ses spectacles ont plus souvent servi de tribunes d'exhibitions folkloriques ou de sensibilisation sociale que de production de véritables représentations artistiques.

Cette observation est globalement vérifiée. Boniface Makponsè, l'une des personnalités éminentes du paysage théâtral de l'époque, reconnaît aussi que sa troupe pratiquait un « théâtre idéologique ${ }^{9}$ ». Le directeur des Muses renchérit en ces termes : « On nous donnait des directives et on produisait. [...] On n'était vraiment pas libre. Du reste, on n'était même pas conscient de la liberté qu'on devait avoir. On nous a moulés dans l'idéologie révolutionnaire. On nous endoctrinait et nous-mêmes on s'endoctrinait ${ }^{10}$. »

Toutefois, une analyse plus minutieuse de ces productions révèle que plusieurs parmi elles, loin d'être «sclérosées », se distinguaient par leur qualité remarquable. La finalité idéologiquement conformiste allait parfois de pair avec une recherche esthétique poussée, notamment dans certains spectacles de l'Ensemble artistique national (Yovo Héluwé ! Akowé Héluwé !, Koyi, towênonblo todo, Zojagé), les créations de l'Ensemble polyvalent Zama Hara (Tomabu, Oni Baba...). En revanche, l'EACE se montrait plus frondeuse, et donc plus libre, dans ses productions. 
À l'image de la FNEB, animée entre autres par des éléments radicaux du Parti Communiste du Bénin, l'EACE affichait ouvertement son indépendance à l'égard des autorités rectorales et du pouvoir politique. Médéhouegnon écrit à juste titre :

La troupe la plus productive et, peut-être aussi, la plus audacieuse de la période révolutionnaire a été sans doute l'Ensemble Artistique et Culturel des Étudiants de l'Université Nationale du Bénin (EACE), créé en 1977. Après des débuts hésitants, l'EACE a progressivement adopté une ligne d'autonomie relative en portant sur la scène des pièces de satire politique et sociale comme Justice à la barre (1983), Le gâteau d'anniversaire (1987), Signez, signez seulement (1988).

Avant 1988, l'EACE avait créé Et la roue de l'histoire tourne (1977), Agbagbé (1977) et Ça brûle (1985). Contrairement aux œuvres des autres troupes, celles de l'EACE étaient pour la plupart des satires politiques féroces, qui dénonçaient les dérives dictatoriales, la corruption ambiante, le népotisme, etc. Si une pièce comme Assez ! de l'Ensemble Zama-Hara stigmatisait les fonctionnaires corrompus, celles de l'EACE situaient à tous les niveaux de l'appareil d'État les responsables de la déroute financière et politique du pays. Personne n'était épargné, même pas les membres de l'Assemblée nationale révolutionnaire, les ministres et le Chef de l'État. Ceux-ci, dans la mesure du possible, affichaient une tolérance condescendante à l'égard des jeunes de l'EACE qu'ils préféraient voir se défouler à travers les créations artistiques plutôt qu'au moyen des marches et manifestations violentes.

Mais la crise économique des années 1980, marquée entre autres par l'effondrement des sociétés d'État, la déroute du secteur bancaire et le cumul des arriérés de salaires dus aux fonctionnaires, va progressivement renforcer les oppositions au régime révolutionnaire. Sur les campus universitaires, les manifestations se multiplient. Toutefois, les répressions violentes des mouvements estudiantins ne faisaient qu'accentuer la verve satirique des comédiens de l'EACE. L'Ensemble a ainsi préparé - et participé, à sa façon -, à la grande vague des manifestations sociales et politiques qui, conjuguées avec une conjoncture internationale provoquée par la chute du mur de Berlin en 1989, vont précipiter la faillite du régime béninois et déboucher, en février 1990, sur l'organisation en Afrique de la première Conférence souveraine des forces vives de la nation.

Les libertés démocratiques ont été retrouvées au lendemain de ces assises. La nouvelle Constitution, adoptée le 11 décembre 1990, reconnaît le multipartisme, la liberté d'expression et de presse et ouvre la voie à l'écono- 
mie de marché. Dans ce nouveau contexte légalement rassurant, les artistes de l'EACE ont multiplié les créations satiriques : Il était une fois (1990), Le Clair-sombre (1990), Le Progrès (1991), Le Temple vert (1993), Le Bal des fous (1994)... Toutes ces pièces étaient des créations collectives. Mais, dès 1995, le rythme de production des spectacles ne sera plus le même au sein de l'Ensemble.

\section{Les années de vaches maigres (1995 à 2009)}

À partir de la seconde moitié des années 1990, le théâtre béninois d'expression française est entré dans une phase de léthargie ${ }^{11}$. Celle-ci s'est accentuée au début des années 2000. L'EACE a, certes, continué à produire mais de manière épisodique. Entre 2007 et 2009, il a présenté au public quelques spectacles comme I've a dream, inspiré du célèbre discours du pasteur Martin Luther King ; Tsunami, une création collective, et Sous le grand kapokier..., d'après un texte de Roger Atikpo ${ }^{12}$.

Dans tous les cas, le ralentissement considérable de la production à cette deuxième phase est manifeste. L'Ensemble a surtout remis sur la scène ses anciennes créations. Par exemple, entre 1995 et 2000, il n’a présenté au Centre culturel français de Cotonou (CCF), un des principaux lieux d'accueil de spectacles de théâtre francophone, que ses anciennes pièces : Il était une fois, Le Clair-sombre, Le Procès, Le Temple vert, Le Bal des fous. Entre 2000 et 2010, il n’a présenté aucun spectacle au $\mathrm{CCF}^{13}$.

Toutefois, à partir de 2010, il y a eu comme un regain de dynamisme au sein de la troupe.

\section{Les années de relance (de 2010 à nos jours)}

Le tableau page suivante est une estimation approximative du palmarès de l'EACE à partir de 2010, d'après les données fournies par les membres du Bureau directeur de la troupe : 
Estimation quantitative de la production théâtrale de l'EACE de 2010 à 2017

\begin{tabular}{|c|c|c|}
\hline Année & Titre des productions théâtrales & Nature/Auteur(s) \\
\hline 2010 & Enquête $n^{\circ} 98$ & $\begin{array}{c}\text { Pot-pourri de textes de Sony Labou } \\
\text { Tansi et de certains membres de } \\
\text { l'EACE }\end{array}$ \\
\hline \multirow[t]{2}{*}{2011} & $3^{\text {e } a l i n e ́ a ~}$ & Création collective \\
\hline & Hwèdosi & Création collective/Ballet \\
\hline $2012(?)$ & Le Chant du bétail & Ballet à thème \\
\hline 2012 & 7 milliards de voisins & $\begin{array}{c}\text { Théâtre/Texte écrit par Giovani } \\
\text { Houansou }\end{array}$ \\
\hline \multirow[t]{3}{*}{2015} & Danses traditionnelles et théâtre & Ballet/Création collective \\
\hline & 7 milliards de voisins & $\begin{array}{c}\text { Théâtre/Texte écrit par Giovani } \\
\text { Houansou }\end{array}$ \\
\hline & L'Être sacré & Ballet à thème/Création collective \\
\hline \multirow[t]{3}{*}{2017} & Les Marginaux & Ballet à thème/Création collective \\
\hline & Il faut battre l'amour quand il est fou & Texte de Jean-Paul Tooh-Tooh \\
\hline & La Mort de la prêtresse & Théâtre/Création collective \\
\hline
\end{tabular}

Source : Données de terrain recueillies par l'auteur, décembre 2017.

Les données ci-dessus sont, bien sûr, partielles et méritent, de l'aveu même des membres du Bureau directeur, d'être vérifiées et complétées. On pourrait leur ajouter d'autres activités théâtrales prévues dans le calendrier de l'Ensemble. Cela permet de dégager une esquisse typologique de la production théâtrale : les productions statutaires (RACU, BORATA, RUITAP...), les grandes productions annuelles, le mini-festival Étincelles et les prestations de services à l'endroit des autorités rectorales et décanales. Avec raison, Euloge Tossou, directeur de l'Ensemble en 2017, conclut : « C'est sur toute l'année que nous créons. L'EACE n'a pas de vacances ni de congés ${ }^{14}$. »

$\mathrm{Au}$ regard de ce rythme intense de productions, plusieurs observations pourraient être faites. Nous remarquons, par exemple, que les ballets et danses occupent de plus en plus une place importante dans la production artistique de l'Ensemble. Entre 1977 et 1995, la troupe estudiantine était surtout connue grâce à ses spectacles de théâtre, très appréciés aussi bien à cause de l'audace du ton que de l'esthétique. Actuellement, les sections « Danses nationales » et «Chorégraphie » semblent avoir le vent en poupe. 
En outre, les « prestations de services » sont de plus en plus régulières et bien recherchées des membres du complexe artistique. On peut, de même, relever une tendance esthétique de plus en plus marquante : c'est la mise en scène de pièces d'auteurs, alors que, entre 1977 et 1995, la création collective était le processus dominant voire exclusif de production théâtrale. Par ailleurs, l'engagement politique s'atténue fortement au profit de spectacles de satire sociale et de critique des mœurs. À titre d'illustrations, Il faut battre l'amour quand il est fou traite du comportement irresponsable de certains hommes politiques qui, imbus de leur pouvoir, sèment la zizanie dans les familles. 3e alinéa évoque l'histoire d'une fille qui a toutes les chances de réussir mais échoue lamentablement. Sept milliards de voisins porte sur l'altérité et la cohabitation en société, etc.

Enfin, tout observateur averti du paysage théâtral peut facilement remarquer, malgré ce dynamisme renaissant de l'EACE, un déclin persistant de sa notoriété comparativement à l'image que la troupe universitaire avait dans l'opinion publique avant 1995. Ces constats incitent à s'interroger sur les facteurs explicatifs de cette situation.

\section{Diagnostic de la crise du théâtre estudiantin}

Plusieurs raisons sous-tendent le déclin de la production et de la notoriété de l'EACE. Sans prétendre à l'exhaustivité, nous pourrions citer la politisation renforcée des associations syndicales estudiantines et l'émiettement du champ culturel estudiantin, l'autocensure et la ruée vers le captage de rente sans oublier l'absence d'une transmission intergénérationnelle des connaissances artistiques.

\section{La politisation accrue des associations syndicales et l'émiettement du champ culturel estudiantin}

L'avènement du multipartisme intégral, consacré par la Constitution béninoise du 11 décembre $1990^{15}$, a entraîné l'apparition d'une kyrielle de partis politiques rivaux. Ceux-ci, très tôt, ont cherché à recruter des membres et n'ont donc pas hésité à ouvrir leurs registres d'adhésion sur les campus universitaires du pays. Il s'est ensuivi une lutte féroce entre les formations rivales pour le contrôle du microcosme estudiantin. La conséquence a été l'éclatement, dès les années 1990, de la FNEB et l'apparition d'associations syndicales rivales : l'Union des étudiants du Bénin (UNEB), proche du régime du président Nicéphore Soglo, premier président élu de l'ère du « renouveau démocratique » (1991-1995), et l'Union nationale des scolaires et étudiants du Bénin, d’obédience communiste. 
Du coup, chaque syndicat s'est doté de sa branche artistique et culturelle. Par exemple, l'UNEB a créé en 1993 l'Union culturelle et artistique des étudiants, laquelle organise aussi une rentrée artistique et culturelle et d'autres activités similaires à celles de l'EACE. Même si la concurrence présente l'atout de créer un environnement de saine rivalité et d'émulation, elle est aussi source de dispersions d'énergies créatrices qui, fédérées et mieux gérées, auraient pu être plus bénéfiques. Cela est d'autant plus vrai que ces structures s'adressent au même public et ont, à quelques exceptions près, les mêmes partenaires techniques et financiers.

\section{Stratégies marketing de captage de rente et autocensure}

Placée sur le même champ culturel, chaque association se doit alors de mettre en œuvre des stratégies pour capter la rente des commandes de prestations de services et, si possible, les subventions éventuelles octroyées par l'administration universitaire. Dans le champ culturel ainsi créé, ces stratégies, comme le montre Bourdieu, « se distribuent entre deux limites qui ne sont, en fait, jamais atteintes, la subordination totale et cynique à la demande et l'indépendance absolue à l'égard du marché et de ses exigences ${ }^{16} \gg$. La tendance est alors forte de produire des spectacles conformes à l'horizon d'attente $\mathrm{du} \ll$ client $\gg$, donc moins critiques. Nous trouvons là une première explication de la dépolitisation ou du désengagement politique du théâtre estudiantin. Les propos ci-dessous d'Euloge Tossou confirment cette analyse :

Il fut une année où on avait présenté pour les autorités un spectacle critique. Elles étaient déçues et ont décidé de ne plus nous appeler. Comme à l'époque, l'EACE était presque seul, ces autorités ont été obligées de revenir vers nous. Mais actuellement, nous sommes trois associations culturelles rivales et, donc, les institutions universitaires peuvent solliciter qui elles veulent ${ }^{17}$.

Mais il y a d'autres raisons relatives, selon les membres du Bureau directeur, au changement du contexte politique et à la censure :

Avant, l'EACE était très critique. Aujourd'hui, précisément depuis les années 2000, nous ne le sommes plus. Pourquoi? D'abord, les temps ont changé. La satire politique frontale avait sa raison d'être quand, en contexte de dictature, il fallait lutter pour l'avènement de la démocratie. Depuis 1990, nous avons la démocratie. Ce n'est donc plus la peine de s'attaquer au système politique, mais au vécu des populations. Aujourd'hui, nous luttons pour la paix. Ensuite, nous craignons les réactions des autorités universitaires. Elles peuvent nous nuire très rapidement si la critique est trop vive. Nous sommes après tout des étudiants! 
Enfin, nous pensons aussi que la satire politique frontale n'est pas forcément la seule et unique voie de résolution des problèmes ${ }^{18}$.

Paradoxale en contexte de démocratie, cette tendance à l'autocensure est assez vive chez plusieurs membres de l'EACE. À l'argument de l'inadéquation du théâtre politique avec la démocratie, il est facile de répliquer que l'avènement de celle-ci n'a pas sonné le glas de tous les problèmes de développement du Bénin, loin de là. Le « renouveau démocratique »a, au contraire, généré d'autres contraintes structurelles majeures sur lesquelles les auteurs dramatiques et les metteurs en scène doivent se pencher.

L'autocensure des étudiants est certainement due, aussi, à l'importance que revêt de plus en plus l'obtention des diplômes pour avoir accès au marché de l'emploi. À l'heure où l'ouverture des écoles doctorales permet aux étudiants de poursuivre leurs études jusqu'à la soutenance de la thèse, et donc de s'astreindre à une collaboration plus étroite et plus courtoise avec les enseignants et les autorités académiques, aucun apprenant ne voudrait courir le risque de perdre le bénéfice d'une inscription onéreuse en master ou en thèse. Cette frilosité à l'égard de la satire politique pourrait également trouver son explication dans l'insuffisance d'une formation artistique solide.

\section{Le sevrage artistique brutal et l'absence d'une transmission générationnelle des connaissances}

Le succès remporté par les créations artistiques de l'EACE entre 1977 et 1995 était incontestablement le fait du génie créateur des membres de la troupe estudiantine et de leur volonté de contribuer au développement de leur pays. Artistes engagés mais sans aucune formation en arts dramatiques, ils n'auraient cependant pas pu atteindre ces résultats s'ils n'étaient épaulés, ne serait-ce qu'au début de leur parcours, par des personnes plus expérimentées. Si l'autodidaxie est la règle, les ateliers de création théâtrale organisés parfois parl'Ensemble étaient des occasions d'apprentissage et de perfectionnement. Paul Ayémona, le père fondateur de l'Ensemble, reconnaît sans ambages que Lazare Houétin, son mentor, a été le premier metteur en scène du complexe. En coulisse, celui-ci apportait sa touche à la création des spectacles.

À son tour, le premier directeur de l'Ensemble, à la fin de son mandat, n'a pas définitivement rompu les relations avec ses anciens camarades. Il a continué à les soutenir à travers ses conseils et l'animation de diverses sessions de formation. Il en a été de même pour la plupart des anciens responsables qui, de 1977 à 1995, se sont succédé à la tête de la troupe estudiantine. Cette chaîne de transmission intergénérationnelle des connaissances, « réduite à l'acquisi- 
tion du savoir-faire traditionnel que se léguaient des lignées d'interprètes ${ }^{19} \gg$, a commencé à se fragiliser avec le lancement de l'émission télévisée à succès Entre-Nous, produite par l'antenne béninoise de l'ONG Africa Obota, mise en place par Paul Ayémona et autres anciens membres de l'EACE dont Lazare M. Sèhouéto, sociologue et actuellement homme politique. Financée par la fondation allemande Konrad Adenauer, cette production télévisée était animée d'un côté par un journaliste de l'Office de radiodiffusion et de télévision du Bénin et, de l'autre, par des artistes recrutés et payés par Africa Obota pour créer des saynètes en guise d'illustrations du thème débattu. Ancien membre actif de l'EACE, Sébastien Davo confirme cette observation :

Être recruté par Afrika Obota pour jouer dans l'un ou l'autre de ses sketches, c'était comme trouver de l'emploi en même temps que se donner une chance de visibilité dans le paysage culturel. Cela a eu comme conséquences, d'une part, la non-disponibilité des anciens membres de l'EACE pour former les nouveaux membres de l'Ensemble et, d'autre part, un désir grandissant de ceux-ci d'abandonner les activités artistiques non lucratives de la troupe universitaire pour venir monnayer leurs talents à Africa Obota ${ }^{20}$.

Brutal a donc été le sevrage artistique et culturel des nouveaux membres de l'Ensemble. Par ailleurs, à l'instar de Marcel Orou Fico, de Théodore Béhanzin, d'Ignace Yêtchénou et d'Orden Alladatin, d'autres anciens membres de l'EACE ont créé leurs propres troupes de théâtre pour continuer à nourrir leurs passions artistiques et profiter des opportunités du marché artistique que sont les commandes de pièces théâtrales, de spots et de téléfilms, les appels à candidatures pour la subvention de projets culturels lancés par le Fonds d'aide à la Culture, l'Union européenne, l'Ambassade de France au Bénin, etc. Non seulement, ils n'ont plus guère le temps pour encadrer leurs jeunes successeurs à l'EACE, mais ils ont recruté des membres dans le vivier artistique originel. L'adhésion à l'Ensemble n'est donc plus un passage obligé pour bon nombre d'étudiants qui, une fois inscrits à l'université, peuvent brûler la politesse aux troupes existantes sur le campus pour faire leurs expériences artistiques ailleurs.

\section{Conclusion}

Le constat relatif à la décadence du théâtre estudiantin béninois est bien réel. Cependant, l'autocensure et la course au captage des rentes n'ont pas été les seuls déterminants de la crise survenue au sein de l'Ensemble Artistique et Culturel des Étudiants à partir de 1995. Il faudrait ajouter, à ces deux raisons, la dispersion des énergies créations due à l'émiettement du paysage théâtral 
estudiantin. Non moins négligeable se révèle l'absence d'une transmission intergénérationnelle des connaissances entre les anciens et les nouveaux membres de la troupe universitaire. L'hypothèse posée au début de cette étude est, donc, incomplète car d'autres facteurs sont à prendre en considération dans l'élucidation de la crise au sein de l'EACE.

La célébration, sur le campus universitaire d'Abomey-Calavi et à Cotonou, du quarantième anniversaire de la troupe universitaire, du 15 au 23 juillet 2017, a été l'occasion de chaudes retrouvailles entre les anciens et les nouveaux membres. À l'unanimité, il a été décidé de créer une Fondation EACE, destinée à impulser une réelle dynamique des activités de l'Ensemble. Il apparaît, cependant, qu'une telle entreprise gagnerait à s'accompagner de mesures destinées à assainir le fonctionnement de la troupe et à sauter l'étau de l'autocensure, frein à la création de pièces audacieuses et novatrices. Il est tout autant indispensable de créer un climat de confiance et de collaboration entre les associations théâtrales estudiantines pour une mutualisation de leurs ressources en vue d'une meilleure animation du paysage dramatique au Bénin.

\section{Notes}

1. Bernard Mouralis, "Pertinence de la notion de champ littéraire en littérature africaine », in Romuald Fonkoua et Pierre Halen avec la coll. de Katharina Städtler (textes réunis par), Les Champs littéraires africains, Paris, Karthala, Halen, 2001, p. 65.

2. Ministère de l'Information et de l'Orientation nationale, « Discours-programme du G.M.R. présenté par le Chef de Bataillon Mathieu Kérékou, chef de l'État, chef du Gouvernement Militaire Révolutionnaire ", in Recueil des discours du camarade résident de la République le lieutenant-colonel Matbieu Kérékou, Cotonou, novembre 1975, p. 15.

3. Arrêté $\mathrm{n}^{\circ}$ 01/PR/MION du 27 mars 1975 « portant réglementation sur les publications à caractère politique ", cité par Adrien Huannou, La littérature béninoise de langue française (des origines à nos jours), Paris, Karthala, 1984, p. 249.

4. Cité par Adrien Huannou, op. cit., p. 248

5. Pour avoir plus d'informations sur le théâtre « pontin » et le palmarès des élèves dahoméens de l'École normale William-Ponty du Sénégal, cf. Pierre Médéhouegnon, $L e$ théâtre francophone de l'Afrique de l'Ouest. Des origines à nos jours (Historique et analyse), préface de Hervé Besancenot, avant-propos du Pr. Jean-Pierre Guingané, Cotonou, éditions CAAREC, 2010.

6. Nous avons proposé une analyse de cette création collective dans un article intitulé : «De Zojagé à Houénoussou : idéologie et esthétique théâtrale en période "révolutionnaire" au Bénin ", Continents manuscrits [En ligne], 13 | 2019, mis en ligne le 15 novembre 2019, consulté le 18 décembre 2019. URL : http://journals.openedition. org/coma/4304

7. Aristide Settin, «Interview avec le directeur Paul Ayémonna à propos des 40 ans de l'EACE : la création de l'EACE n'est pas le fait de penser et de décider d'un individu ; mais la résultante de plusieurs circonstances ", Le Canal de l'Art, journal de l'Ensemble 
Artistique et Culturel des Étudiants, parution spéciale des 40 ans, Abomey-Calavi, 2017, p. 5. NDA : Note de l'Auteur de l'article. Selon Paul Ayémona, l'idée de création de l'Ensemble remonte à décembre 1976, mais c'est au cours de l'année suivante que la troupe a été portée sur les fonts baptismaux. À l'évidence, et au regard des problèmes de formulations qui émaillent l'entretien, l'auteur de l'article, un dilettante en journalisme, n'a pas su bien réussir le traitement des propos de M. Paul Ayémona, un intellectuel assermenté et un médecin très connu dans le pays.

8. Pierre Médéhouegnon, Innovations et créations dans le théâtre francophone de l'Afrique de l'Ouest (État des lieux et analyse de la dramaturgie en Côte d'Ivoire, au Burkina-Faso, au Togo et au Bénin, de 1970 aux années 2000), Thèse de doctorat d'État, Université d'Abomey-Calavi, 2009, p. 177.

9. Boniface Makponsè, cité par Fernand Nouwligbèto, Théâtre béninois : logiques marchandes et enjeux esthétiques, Cotonou, CIREF, 2017, p. 40-41.

10. Op. cit., p. 41.

11. Pour comprendre les raisons de cette léthargie du paysage théâtral dans son ensemble, lire : Pierre Médéhouegnon, «Le théâtre béninois des années 2000 : une dramaturgie en quête de repères ? ", in Adrien Huannou (textes réunis et présentés par), Repères pour comprendre la littérature béninoise, Cotonou, éditions CAAREC, 2008, p. 29-52 ; Fernand Nouwligbèto, op. cit., p. 60-82.

12. L'inexistence d'une section de documentation et d'archivage au sein de l'Ensemble rend particulièrement difficile l'accès à des données précises et fiables sur cette période.

13. Fernand Nouwligbèto, Le Théâtre béninois d'expression française de 1990 à 2010: logiques marchandes et enjeux esthétiques, Thèse de doctorat unique, Université d'AbomeyCalavi, 2012, p. 117.

14. Entretien avec Euloge Tossou et les autres membres du Bureau directeur de l'EACE, Campus universitaire d'Abomey-Calavi, $1^{\text {er }}$ décembre 2017.

15. La révision de cette Constitution, intervenue le $1^{\text {er }}$ novembre 2019, a maintenu les principes du multipartisme et de la liberté d'expression.

16. Pierre Bourdieu, Les Règles de l'art. Genèse et structure du champ littéraire, Paris, Seuil, 1998 ( $2^{\mathrm{e}}$ édition), p. 235.

17. Entretien avec Euloge Tossou et les autres membres du Bureau directeur de l'EACE, Campus universitaire d'Abomey-Calavi, $1^{\text {er }}$ décembre 2017.

18. Ibid.

19. Michel Pruner, La Fabrique du théâtre, Paris, Armand Colin, 2010, p. 150.

20. Entretien avec Sébastien Davo, ancien membre de l'EACE, Campus universitaire d'Abomey-Calavi, 2 décembre 2017. 